\title{
Homogenization of Voxel Models using Material Mixing Formulas
}

\author{
Tushar Singh ${ }^{1}$, Mladjen Stevanetic ${ }^{2}$, Marija Stevanovic ${ }^{3}$, Branko Kolundzija ${ }^{4}$ \\ ${ }^{1}$ WIPL-D, Belgrade, Serbia, tushar.singh@wipl-d.com \\ ${ }^{2}$ WIPL-D, Belgrade, Serbia, mladjen.stevanetic@wipl-d.com \\ ${ }^{3}$ Faculty of Electrical Engineering, University of Belgrade, Belgrade, Serbia, mnikolic@etf.rs \\ ${ }^{4}$ WIPL-D, Belgrade, Serbia, branko.kolundzija@wipl-d.com
}

\begin{abstract}
In this paper, we develop a procedure for simplifying highly inhomogeneous numerical phantoms based on dielectric mixing formulas. Numerical phantoms are extremely important in designing microwave imaging systems and algorithms. However, most of the realistic phantoms, typically obtained from magnetic resonance imaging (MRI) or computerized tomography (CT) scans are unsuitable for realtime analysis due to unlikely requirements for computational power and long processing time. Hence, it is of great importance to simplify such models without sacrificing the accuracy of the electromagnetic analysis. Here, we obtain simplified models by replacing a group of voxels by an effective permittivity computed by means of Looyenga and Lichtenecker methods. To assess the accuracy of the homogenized models with different resolutions, we compare their radar cross sections as well as transmissions between the antennas placed in their vicinity.
\end{abstract}

Index Terms-Antenna, Microwave Imaging, Breast Phantom, Litchnecker, Looyenga, WIPL-D.

\section{INTRODUCTION}

Over the last decade, there has been a significant interest in using the microwaves for breast-cancer detection [1]. Soon after, the list of potential applications of microwave imaging in medical diagnostics included bone imaging, stroke detection, etc. [2]. The main advantages of microwave imaging systems are cost-effectiveness and portability, compared to the conventional technologies such as magnetic resonance imaging (MRI) and computed tomography (CT). Although MRI and CT are still considered as gold standards in clinical diagnosis, they have several drawbacks such as high cost, large size, and ionizing radiation.

Phantoms play an important part in the design of microwave imaging systems [3]. They are equally important in hardware design, i.e., when optimizing the performance of the antennas, as well as in software design, i.e., when testing the imaging algorithms. In order to study the interaction between the human tissues and electromagnetic waves, many phantoms have been developed, e.g., [3]-[5].

In contrast to physical phantoms, numerical phantoms can readily capture the complex heterogeneous interior of human tissues. In this paper, we use the realistic breast phantom derived from the series of T1-weighted Magnetic Resonance Images (MRI), available at the online repository [6]. The proposed numerical phantom captures detail about structural complexities and dispersive dielectric distribution of tissues inside the breast. With its $0.5 \mathrm{~mm}$ resolution, voxel model is valuable due to its high-fidelity; however, it is difficult to use it in real time applications due to the large number of voxels. Namely, each voxel has a different permittivity value so the numerical implementation of such model requires a large number of unknowns.

In this paper, we propose a homogenization method, based on Lichtenecker and Looyenga mixing formulas [7]-[10] to decrease the number of unknown coefficients in the numerical analysis of such a model. By homogenization, we mean assigning some average permittivity to a group of heterogeneous voxels, while keeping the electromagnetic response almost intact. To validate the proposed method, we compute the scattering from the homogenized model as well as the transmission between antennas placed in the vicinity of the homogenized model. In this paper, we conduct uniform homogenization.

The paper is organized as follows. After the Introduction, in Section II we give a short description of the utilized voxel model. In Section III, we describe the homogenization method and the mixing formulas. Section IV presents a brief analysis of the obtained numerical results and demonstrates the effectiveness of the homogenization method in reducing the number of unknowns without sacrificing the accuracy. Finally Section V concludes the paper.

\section{VOXEL MODEL OF BREAST}

In this paper, we use a breast voxel model from the online repository that comprises the database of anatomically realistic numerical breast phantoms [6]. The phantom was derived from an MRI image of a patient. It is a threedimensional (3D) grid of voxels where each voxel is a cube of the size $0.5 \mathrm{~mm} \times 0.5 \mathrm{~mm} \times 0.5 \mathrm{~mm}$. Besides breast tissues, the model also contains a skin-layer of the thickness $1.5 \mathrm{~mm}$, subcutaneous fat-layer of the thickness $1.5 \mathrm{~cm}$, and a muscle chest wall of the thickness $0.5 \mathrm{~mm}$. There are three different repository text-files in the database, named as "breastInfo.txt", "mtype.txt", and "pval.txt". The 3D numerical model is computed with the help of those three files. The first file "breastInfo.txt" gives the basic information about the grid size in the $3 \mathrm{D}$ geometry, as $s_{1}, s_{2}$, and $s_{3}$ are the number of the voxels along the $x, y$, and $z$-axis, respectively. The second file "mtype.txt" provides information about the tissue type for each voxel in the grid. As shown in Table I, one value is assigned for each tissue. The third file "pval.txt" describes the 
variation of the electrical properties of the same type of tissues.

TABLE I. TISSSUE TYPES AND CORRESPONDING MEDIA NUMBER

\begin{tabular}{|l|c|}
\hline \multicolumn{1}{|c|}{ Tissue Type } & Media Number \\
\hline Immersion Medium & -1 \\
\hline Skin & -2 \\
\hline Muscle & -4 \\
\hline Fibroconnective/glandular -1 & 1.1 \\
\hline Fibroconnective/glandular -2 & 1.2 \\
\hline Fibroconnective/glandular -3 & 1.3 \\
\hline Transitional & 2 \\
\hline Fatty -1 & 3.1 \\
\hline Fatty -2 & 3.2 \\
\hline Fatty -3 & 3.3 \\
\hline
\end{tabular}

The assigned permittivities were obtained from the large-scale study of ultrawideband microwave dielectric properties of normal breast [11] and were analytically described by means of single pole Cole-Cole formula.

To illustrate the level of heterogeneity of tissues within the considered breast phantom, we show a permittivity distribution within a small cube of the size $8 \times 8 \times 8$ in voxels (i.e., $4 \mathrm{~mm} \times 4 \mathrm{~mm} \times 4 \mathrm{~mm}$ ) The red color indicates higher values of dielectric constant and the blue color indicates the lower values of the dielectric constant. (In general, the relative permittivity in the model ranges from less than five for fatty tissue up to sixty for fibro-glandular tissue.) Hence, computing the electromagnetic response of the whole model requires high computational power and long processing time due to the large variability of tissue properties. Therefore, a homogenization process is required to simplify the model and make it suitable for numerical analysis.

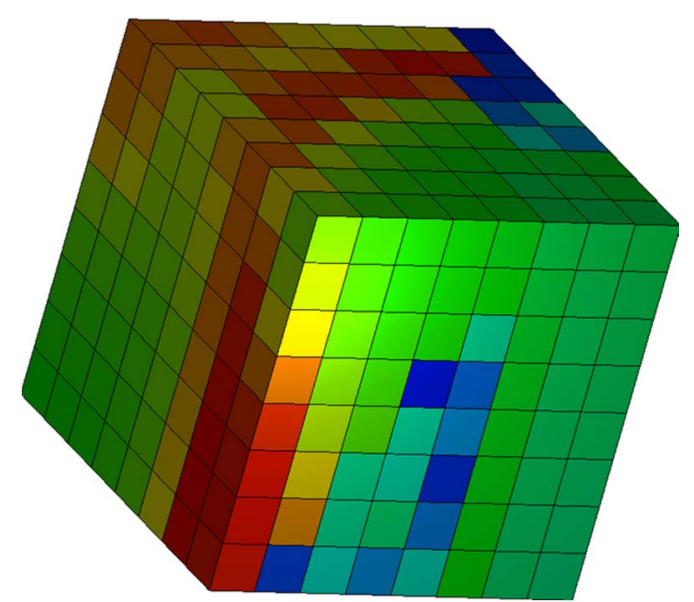

Fig.1. The permittivity distribution with a cube of the size $8 \times 8 \times 8$ (in voxels)

\section{HOMOGENIZATION METHOD}

In this paper, we propose a homogenization method for highly inhomogeneous tissues, which provides an effective dielectric constant for groups of voxels. We assume that the voxels are grouped together in large cubes. The size of the large cube is defined by the integer $n$, which represents the number of the original voxels along each axis of the cube. For example, $n=8$, means that the total number of the voxels in the cube is 512. The size of the large cube is same for the whole phantom. In Fig. 2, we show the equivalent breast model obtained after the homogenization.

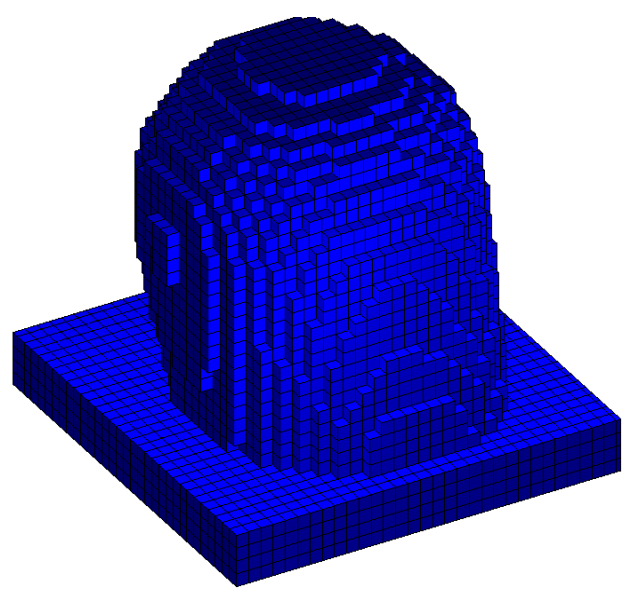

Fig. 2. The homogenized breast model with the size of the cube $n=8$.

Initially, the effective permittivity of large cubes was determined by the standard averaging procedure

$$
\varepsilon_{\mathrm{eff}}=\frac{1}{N} \sum_{i=1}^{N} \varepsilon_{i}
$$

where $N$ is the total number of the voxels in one large cube and $\varepsilon_{i}$ is the permittivity of the $i^{\text {th }}$ voxel. However, the effective permittivity obtained in this way did not provide optimal results. Namely, even for small number of $N$ scattering from the homogenized model significantly differed from the original model. In order to optimize the effective permittivity, different mixing formulas were studied [7]-[10]. The best results were obtained by means of Looyenga and Lichtenecker equations described below.

\section{A. Lichtenecker}

The effective permittivity of a dielectric mixture given by Lichtenecker formula [9] is

$$
\ln \left(\varepsilon_{\mathrm{eff}}\right)=\frac{1}{N} \sum_{i=1}^{N} \ln \left(\varepsilon_{i}\right)
$$

where $N$ is the total number of different dielectrics and $\varepsilon_{i}$ is the permittivity of the $i^{\text {th }}$ dielectric. Here, $N$ is the total number of voxels in one large cube and is the permittivity of the $i$ th voxel. The Lichtenecker formula has been used to describe a wide range of biological materials such as human blood [9]. Typically, the number of the components in a mixture was two or three. However, the number of the components in the considered problem was significantly larger, e.g., for $n=8$, the total number of components was 
512. Hence, we had to adapt the formula for such a large number of components.

\section{B. Looyenga}

The effective permittivity of a mixture, by means Looyenga formula [10], [11] is given by

$$
\varepsilon_{\mathrm{eff}}^{1 / M}=\frac{1}{N} \sum_{i=1}^{N} \varepsilon_{i}^{1 / M},
$$

where $M=1,2,3$ is a dimensionless factor [11]. In this work, we used $M=3$.

\section{NUMERICAL RESULTS}

To assess the impact of the homogenization, we computed the radar cross section (RCS) of the breast models obtained for different values of the parameter $n$. The simulations were performed at $1 \mathrm{GHz}$, using the WIPL-D software [12]. Fig. 3 and Fig. 4 show the RCS of the homogenized breast models obtained by means of Lichtenecker and Looyenga mixing formula, respectively. In the simulations, the smallest value of the parameter $n$ was 12 . The corresponding side length of the large cube was $6 \mathrm{~mm}$. The largest considered value was $n=32$, corresponding to the cube of the side length $16 \mathrm{~mm}$.

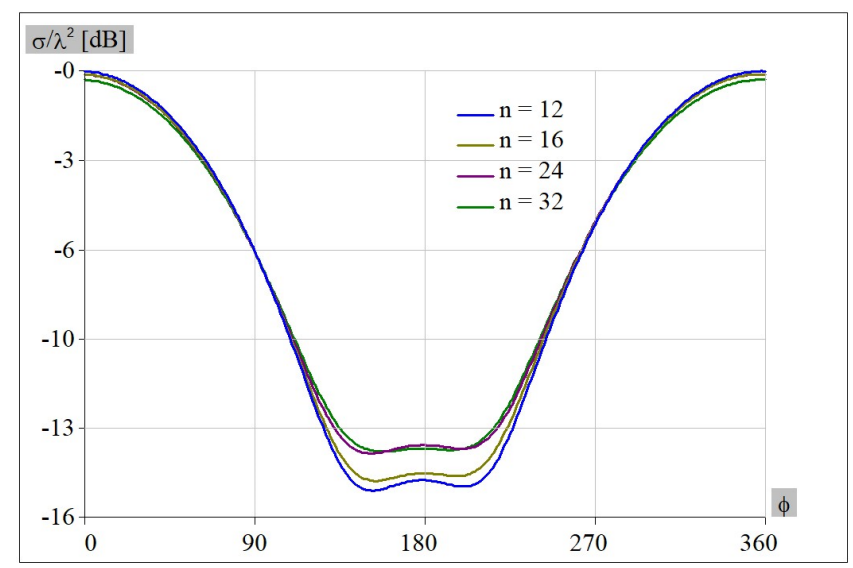

Fig. 3. Normalized RCS of the homogenized breast model obtained by means of Lichtenecker formula with $n=12, n=16, n=24$ and $n=32$, computed at $1 \mathrm{GHz}$.

Both mixing formulas yielded similar results for the RCS. The results obtained for $n=12$ and $n=16$ were almost the same. Discrepancy occurs for angles close to $\phi=180^{\circ}$, when $n=24$ and $n=32$.

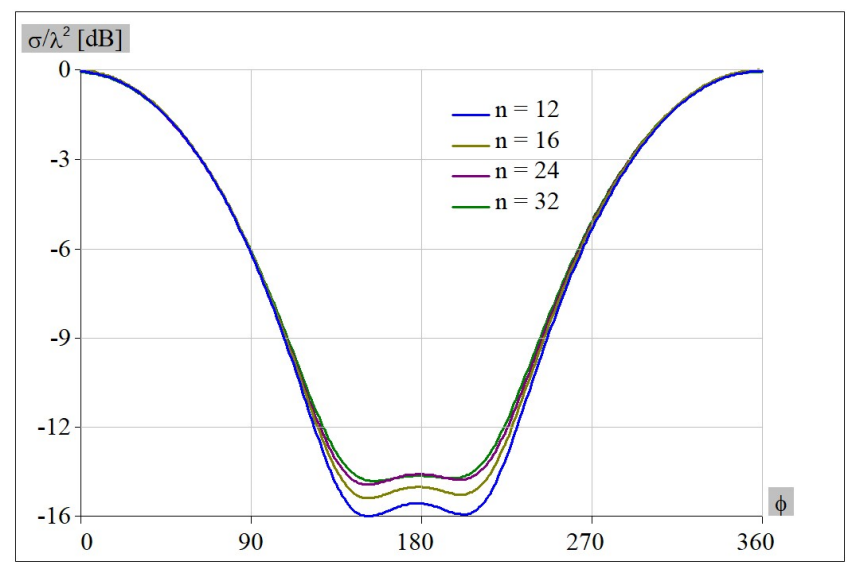

Fig. 4. Normalized RCS of the homogenized breast model obtained by means of Looyenga formula with $n=12, n=16, n=24$ and $n=32$, computed at $1 \mathrm{GHz}$.

To further explore the effect of the homogenization, we computed the transmission coefficient between a pair of dipole antennas placed at the opposite sides of the breast model, as shown in Fig. 5. In Fig. 6, we show the results for the transmission coefficient $\left(s_{21}\right)$ obtained using the Looyenga mixing formula for $n=12,16,24$, and 32. The corresponding frequency range was from $0.9 \mathrm{GHz}$ to $1.1 \mathrm{GHz}$. As in the case of RCS, there was an excellent agreement between the results obtained for $n=12$ and $n=16$. However, the results obtained for $n=32$ significantly differed from the results obtained for $n=12,16,24$, in particular in the lower part of the selected frequency bandwith. Similar results were obtained using the Lichtenecker formula.

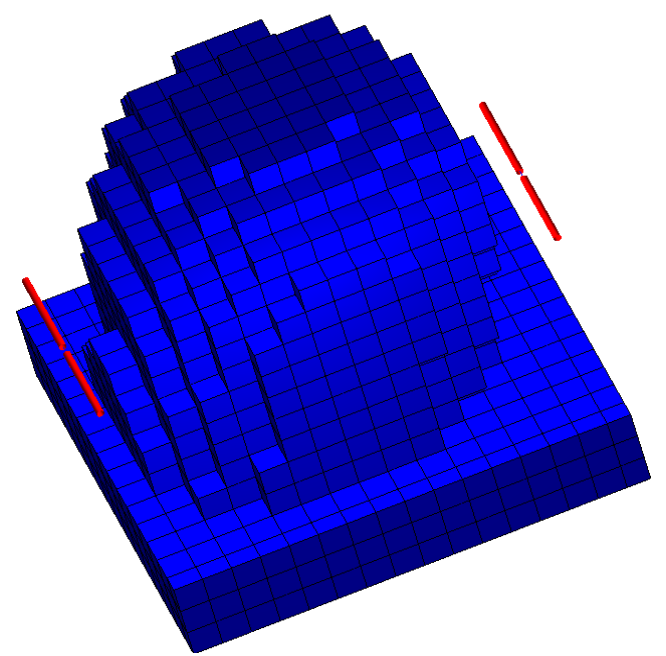

Fig. 5. Two dipole antennas placed at opposite sides of the breast model obtained for $n=16$ 


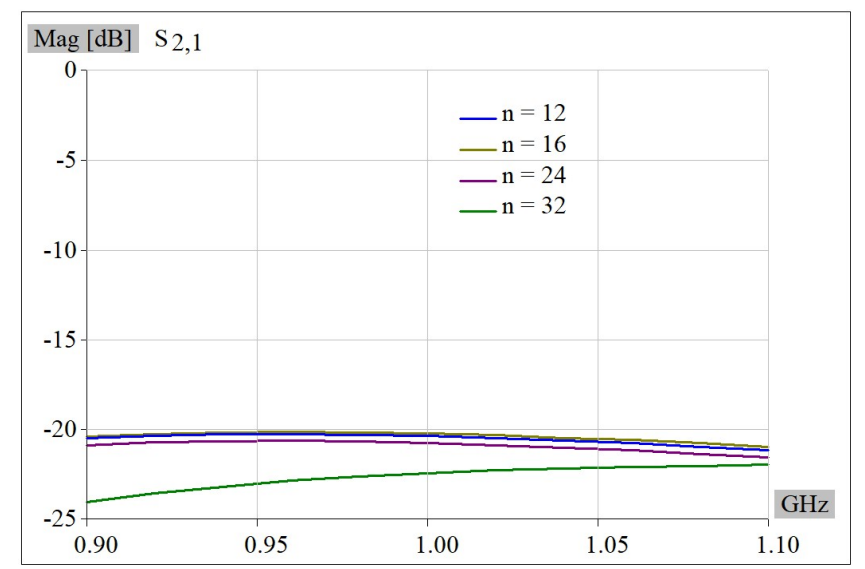

Fig.6. Transmission coefficient between two dipoles placed at the opposite sides of the homogenized breast model obtained by means of Looyenga

formula with $n=12, n=16, n=24$ and $n=32$, computed at $1 \mathrm{GHz}$.

The purpose of the homogenization is to reduce the computational time. Hence in Tab. II, we show the number of the unknowns for each model.

TABLE II NUMBER OF UNKNOWNS OF HOMOGENIZED MODELS WITH DIFFERENT RESOLUTIONS

\begin{tabular}{|r|c|c|}
\hline $\mathrm{n} n$ & $\begin{array}{c}\text { Homogenized cube } \\
\text { side length }\end{array}$ & $\begin{array}{c}\text { Number of unknowns } \\
\text { in the WIPL-D model }\end{array}$ \\
\hline 12 & $6 \mathrm{~mm}$ & 26682 \\
\hline 16 & $8 \mathrm{~mm}$ & 13812 \\
\hline 24 & $12 \mathrm{~mm}$ & 5212 \\
\hline 32 & $16 \mathrm{~mm}$ & 2708 \\
\hline
\end{tabular}

V. CONCLUSION

In this paper, we proposed the method for the reduction of the level of complexity of breast phantoms using Lichtenecker and Looyenga mixing formulas. By computing the RCS and transmission between two antennas, we estimated what is optimal trade-off between the numerical efficiency and the accuracy of the electromagnetic model. At $1 \mathrm{GHz}$, the significant reduction of quality appeared for cubes larger than $12 \mathrm{~mm}$.

\section{ACKNOWLEDGMENT}

This work was supported by the EMERALD project funded from the European Union's Horizon 2020 research and innovation programme under the Marie SkłodowskaCurie grant agreement No. 764479 [13].

\section{REFERENCES}

[1] N. K. Nikolova, "Microwave Imaging for Breast Cancer," in IEEE Microwave Magazine, vol. 12, no. 7, pp. 78-94, Dec. 2011.

[2] M. Persson, A. Fhager, H. Trefna, Y. Yinan, T. McKelvey, G. Pegenius, J.-E. Karlsson, and M. Elam, "Microwave-based stroke diagnosis making global prehospital thrombolytic treatment possible," IEEE Trans.Biomed. Eng., vol. 61, pp. 2806-2817, Nov 2014.

[3] R. Ortega-Palacios, L. Leija, and A. Vera, "Tumor Breast Phantom vs Breast Phantom Microwave Ablation: Thermal Experimentation and
Electric Property Measurements”, 2012 9th International Conference on Electrical Engineering, Computing Science and Automatic Control (CCE), 26-28 Sept. 2012.

[4] Nadine Joachimowicz, Christophe Conessa, Tommy Henriksson, and Bernard Duchêne, "Breast Phantoms for Microwave Imaging", IEEE Antennas and Wireless Propagation Letters, Vol. 13, 2014.

[5] Luz Maria Neira, R. Owen Mays, and Susan C. Hagness, "Development and Application of Human Breast Phantoms in Microwave Diagnostic and Therapeutic Technologies," 2016 38th Annual International Conference of the IEEE Engineering in Medicine and Biology Society (EMBC), 16-20 Aug. 2016

[6] E. Zastrow, S. K. Davis, M. Lazebnik, F. Kelcz, B. D. Van Veen, and S. C. Hagness, "Database of 3D Grid-Based Numerical Breast Phantoms for use in Computational Electromagnetics Simulations," Department of Electrical and Computer Engineering University of Wisconsin-Madison.

[7] Greffe, J. L. and C. Grosse, "Static permittivity of emulsions," Progress In Electromagnetics Research, Vol. 06, 41-100, 1992.

[8] Ray Simpkin, "Derivation of Lichtenecker's Logarithmic Mixture Formula From Maxwell's Equations," IEEE Transactions on Microwave Theory and Techniques, Vol. 58, No. 3, March 2010.

[9] S.O. Nelson, "Measurement and Computation of Powdered Mixture Permittivities," Proceedings of the 17th IEEE Instrumentation and Measurement Technology Conference, DOI: 10.1109/IMTC.2000.848661.

[10] Kimmo Kalervo Kärkkäinen, Ari Henrik Sihvola, and Keijo I. Nikoskinen, "Effective Permittivity of Mixtures: Numerical Validation by the FDTD Method," IEEE Transactions on Geoscience and Remote Sensing, Vol. 38, No. 3, May 20001303.

[11] Mariya Lazebnik, Leah McCartney, Dijana Popovic, Cynthia B Watkins, Mary J Lindstrom, Josephine Harter, Sarah Sewall, Anthony Magliocco, John H Booske1, Michal Okoniewski and Susan C Hagness, "A large-scale study of the ultrawideband microwave dielectric properties of normal breast tissue obtained from reduction surgeries," Phys. Med. Biol. 52 (2007) 2637-2656.

[12] WIPL-D Pro CAD 2019, WIPL-D d.o.o, Belgrade, 2019

[13] Full Wave Modelling for EM Medical Devices. Retrieved from http://www.msca-emerald.eu/research/wp3/task 6 